\title{
Design Scheme of Remote Monitoring System Based on Qt
}

\author{
Dawei $\mathrm{Xu}^{1, a}$, LiPing Yang ${ }^{2}$ and Li Jiang ${ }^{1}$ \\ ${ }^{1}$ School of Electronic and Information Engineering, Changchun University, Changchun, 130022, China \\ ${ }^{2}$ College of Computer Science and Technology, Changchun University, Changchun, 130022, China
}

\begin{abstract}
This paper introduces a design scheme of remote monitoring system based on Qt, the scheme of remote monitoring system based on S3C2410 and Qt, with the aid of cross platform development tools Qt and powerful ARM platform design and implementation. The development of remote video surveillance system based on embedded terminal has practical significance and value.
\end{abstract}

This paper introduces a design scheme of remote monitoring system based on Qt. The remote monitoring system designed in this paper is based on the platform of cross platform development tools Qt and powerful ARM platform. Its practicability and good stability and ease of operation gradually become an indispensable part of embedded devices. The development of remote video surveillance system based on embedded terminal has practical significance and value. According to the principle of the Qt and S3C2410 remote video monitoring system, the remote monitoring system based on Qt is designed and realized.

\section{Overall Design Scheme}

\subsection{Design Main Module}

Remote video surveillance system divides into two parts: software system and hardware system and system software, which includes using QT software development of man-machine interface in Windows system, the third party of OpenCV library, the hardware system including S3C2410 board, servfox acquisition tool (using a Linux system compilation), and Vimicro camera. The overall module design block diagram is shown in figure 1 .

Qt human machine interface in this system is a part of the software, mainly through the Qt interface to deal with the video from the terminal [1].

OpenCV in this system is also part of the software, the main let Qt import OpenCV library files, through the OpenCV provided by Qt to achieve a number of interfaces, so that the smooth display of video images from the terminal.

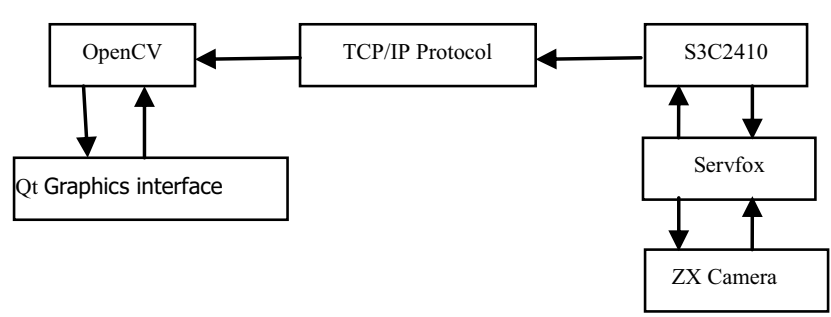

Figure 1. System design block diagram

TCP/IP protocol is the most popular and commonly used network transmission protocol in this system, through the TCP/IP protocol to connect the software part of the system and the hardware of the system, and the video information is transmitted to the client computer through the network cable, as long as the network address of the two terminal can be transmitted through ping.

S3C2410 board in the system in the hardware part, and is also the core of the hardware part, through the S3C2410 port of the USB board connected to the camera, receiving video information collected from the camera, the video information will be collected through the cable to send[2][3].

Servfox belongs to the hardware part of this system, it is mainly through the transplant to the S3C2410 platform, after the detection of the camera, through the command to start the servfox to complete the task of collecting video information, and on the specific video information in the hardware part of the S3C2410 processing[4].

$\mathrm{ZX}$ camera in the hardware part is connected to the S3C2410 port of USB, complete video image acquisition $[5]$.

\subsection{System Design Overall Structure}

\footnotetext{
${ }^{\text {a }}$ Corresponding author: 24003908@qq.com
} 
As shown in Figure 2, the overall structure is divided into two parts of the terminal and the client, the client is mainly Qt the man-machine interface, through the call third party library to achieve the display, while the specific camera, closed, the start of the operation is to complete the man-machine interface [6].

In the main terminal is $\mathrm{S} 3 \mathrm{C} 2410$ board, around the board connection $\mathrm{ZX}$ camera to capture the video terminal, by transplanting to card operating system servfox tools to complete the video stream transmission. The connection of the terminal and the client is mainly through the cable to connect the terminal to send video information, the client accepts the video information at the same time, so that it becomes a whole[7][8].

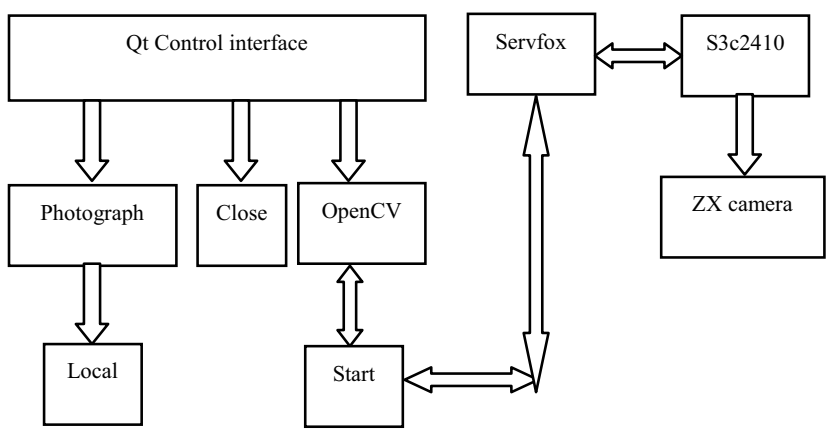

Figure 2. Overall system block diagram

\section{System Hardware Design}

\subsection{Power Interface Circuit}

The circuit diagram of the board's power interface is shown in Figure 3:

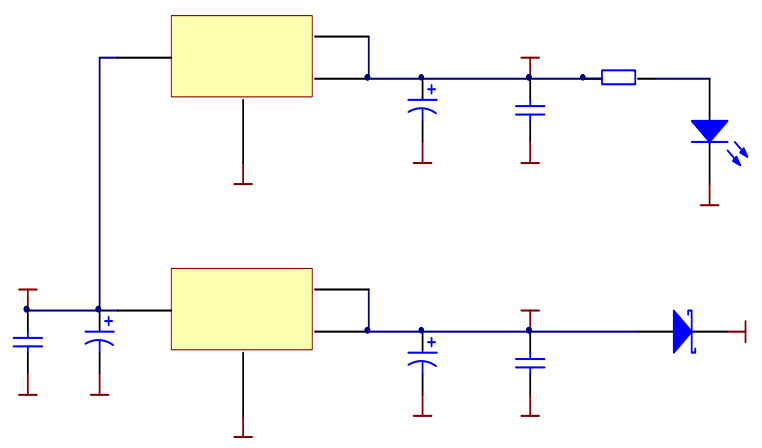

Figure 3. Power interface circuit diagram

\subsection{USB Interface Circuit}

The circuit diagram of the USB interface is shown in Figure 4:
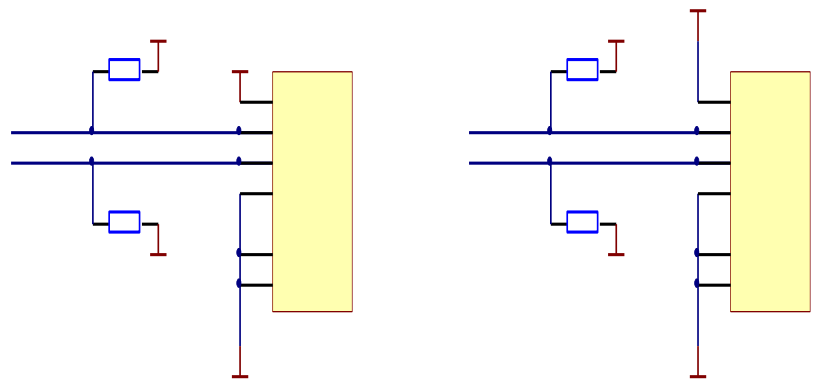

Figure 4. USB interface circuit diagram

Boards have a USB master port and a USB from the mouth, the circuit is mainly on the drop resistance. USB can be detected from the mouth of the cable insertion action, and can cause INT10 interrupt. In this design is mainly used in the USB main port to connect the camera.

\subsection{RS232 Serial Circuit}

The circuit diagram of the serial port interface is shown in Figure 5:
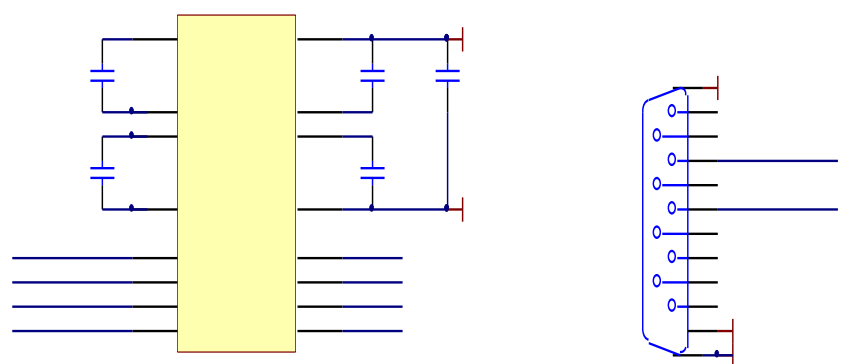

Figure 5. Serial interface circuit diagram

UART1 UART0 and S3C2410, MAX3232 chip to do TTL-RS232 level conversion. Which UART0 corresponding to the RS232 serial port with the DB9 lead, easy debugging. UART1 corresponding RS232 serial port from the expansion of the lead. There are also UART2 (TTL level) on the socket. In this design mainly uses the DB9 socket to connect to the computer, such convenient debug board.

\subsection{Board NandFlash Chip}

The schematic diagram of the NandFlash chip is shown in Figure 6.

K9F1208 is the Nan Flash chip of the Samsung Corp, while the S3C2410 board has a Nand controller, so the K9F1208 is directly connected to the S3C2410 and can be guided from the NandFlash. 


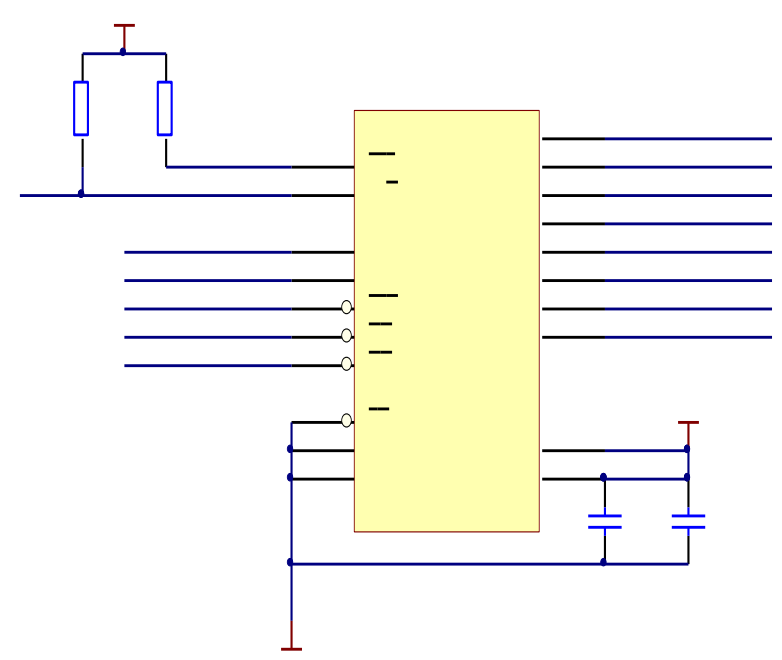

Figure 6. Schematic diagram of the NandFlash

\section{$2.5 \mathrm{ZX}$ camera}

ZX zc301 model of the camera is used in the terminal, the reason for choosing this model of camera is because S3C2410 already supports this model, do not need to re configuration, just need the S3C2410 in loading the camera can be driven, the drive can be found in the CentOS system, specific directory is /upStar2410/kernel/linux/drivers/media/video/zc0301, this directory zc0301.ko is driven will copy it to S3C2410, execute income zc0301.ko can.

\section{System Software Design}

The video surveillance system is composed of the server program and the client program. Server (server) is a network server program based on the Linux system environment. V412 (for Linux II video) is used to control and access the network camera (USB camera) and capture the video data captured by the camera, then compressed by Huffman encoding, and then packaged into HTTP data frame and sends the HTTP protocol to the client.

Because the video surveillance system of the server (server) is transmitted through the HTTP protocol, so the system client has no platform limits, so long as the platform supports HTTP1.0 can access the system services. Moreover, the system's services can be accessed directly through the web browser (Google, Chrome Firefox browser support for the best). In order to improve the transmission efficiency, we also need to carry out the software development of the client, because the client has no platform requirements, that is to achieve cross platform, so in Windows, Linux, Android and other platforms can be achieved. The design of the video monitoring system is mainly to achieve simple client software with Linux as an example of QT, and also can use the browser to access, both at the same time to achieve the $\mathrm{C} / \mathrm{S}$ architecture and $\mathrm{B} / \mathrm{S}$ architecture of the network video surveillance system.
The overall structure of the video surveillance system is shown in Figure 7.

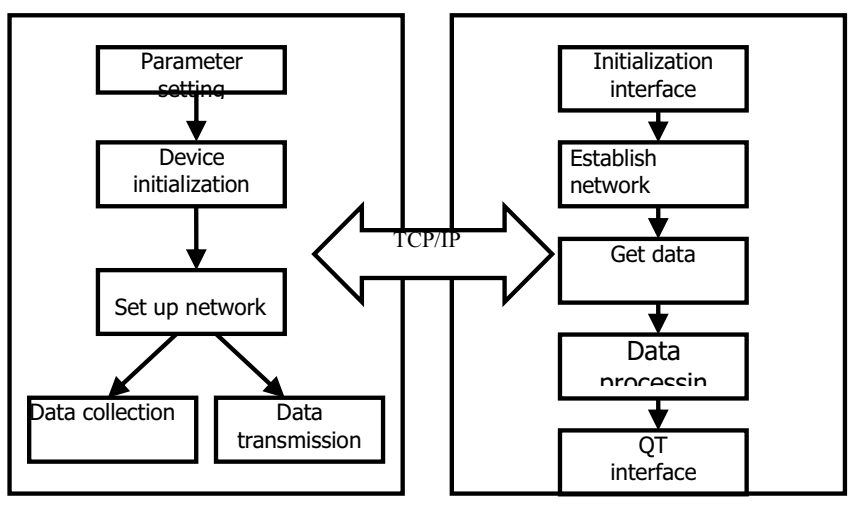

Figure7. Structure diagram of video surveillance system

Server is responsible for the collection of video data acquisition; acquisition can be achieved through the v412 programming, a detailed API development documentation, as well as the relevant information on the v412 programming.

Client is primarily responsible for the reception of the video data through QT programming will be rendered out. Mainly used in QT signal and slot mechanism, as well as graphics events, QT is the use of $\mathrm{C}++$ programming, and it is a set of its own library can achieve cross platform, which also solves the cross platform characteristics mentioned above.

There are connected Server and Client is the use of Socket network server, which is mainly used in Linux environment network programming. The construction of the network is based on the HTTP protocol, the TCP/IP protocol, the server side of the video data is encapsulated into the HTTP data frame, and then use the HTTP protocol to transmit video data to the client. Because it is a real-time transmission, so it is used in multi thread, so that each client is independent each other, each thread will execute a dead cycle, a steady stream of video data to the client.

\section{Conclusion}

In this paper, the design of the remote monitoring system based on S3C2410 and Qt is designed. The system is strictly according to the above process. The video is transmitted in the network. The rest of the operation is mainly concentrated on the interface between human and computer. The remote monitoring system designed in the scheme not only can display the video state of the terminal in time, but also can capture any time. And it can also be used in many situations: the factory warehouse management, mobile phone, PDA, and other small or handheld embedded devices.

\section{References}


1. South J, Blass B (2001) the future of modern genomics. Blackwell, London1 ABRAMSON, S. and LEVI, E., "System and method for determining the location of a machine,"Sept. 26 2007. US Patent App. 11/861,978.

2. ANGERER, C., LANGWIESER, R., and RUPP, M., "Direction of arrival estimation by phased arrays in RFID," in Workshop on RFID Technology, 2010.

3. JAIN, A. and KEMP, C. C., "EL-E: An Assistive Mobile Manipulator that Autonomously Fetches Objects from Flat Surfaces," Autonomous Robots, 2010.

4. PC21 31/2/4/6/8 User manual. Rev. 02. 25 July 2006.

5. Dominique Paret, RFID and contactless smart card applications, Hoboken, NJ: Wiley, 2005.

6. Lin Jin. The application and development of network video surveillance technology [D]. Beijing: Beijing University of Posts and Telecommunications .2007.6, 7:38.

7. Liu Bin. Design of video acquisition module based on embedded system [D]. Xi'an Electronic and Science University, 2006, 5, 3:38.

8. Liu Fuqiang. Development and application of digital video surveillance system [M]. Beijing: Mechanical Industry Press, 2003. 